\title{
Lisis de adherencias epidurales
}

a existencia de adherencias en el espacio epidural, se ha destacado siempre como una de los desencadenantes del síndrome de dolor radicular. Esto es más evidente en el caso del síndrome postlaminectomía donde la aparición de fibrosis siempre ha sido un de los factores que han intentado explicar la clínica de dolor radicular que lo acompaña, y menos evidentes en otros cuadros de dolor, como la estenosis de canal o las discopatías lumbares que se acompañan de dolor irradiado. Sabemos que las sustancias contenidas en el núcleo pulposo pueden desencadenar la aparición de fibrosis, cuando se ponen en contacto con el espacio epidural y las raíces nerviosas en él alojadas (1), por tanto la enfermedad discal degenerativa y las discectomías favorecen este mecanismo. Desconocemos el papel que juega en este proceso la inflamación acompañante en el espacio epidural y en las raíces nerviosas y porqué no todos los pacientes que tienen fibrosis tienen dolor. La lisis de adherencias, técnica percutánea descrita por Gabor Racz en el año 89 y que posteriormente han continuado otros autores especialmente Manchikanti con diferentes variaciones en la duración del tratamiento entre dos o un día (2) y la utilización de diversas medicaciones como la hialuronidasa, el suero salino hipertónico y los esteroides. Las revisiones de la evidencia realizadas en los últimos años demuestran la evidencia fuerte respecto a la mejoría a corto plazo que produce la técnica en pacientes con adherencias epidurales y dolor radicular, siendo la evidencia a largo plazo menos fuerte (3), siendo débil la evidencia en la utilización de hialuronidasa y el suero salino hipertónico. En cuanto a las complicaciones, se han descrito en la literatura algunas de repercusión importante (4-6) si bien en la revisión anteriormente mencionada, no parecían existir complicaciones graves. Parece conflictiva la decisión de utilizar suero fisiológico hipertónico, sobre todo en la región cervical o torácica, donde las complicaciones neurológicas pueden ser mucho más graves que en la zona lumbar, sobre todo cuando el nivel de evidencia de su utilidad es débil. En los últimos años se ha comenzado a realizar la técnica de epiduroscopia, que despierta más expectativas, sobre todo cuando se realizan tratamientos de liberación de adherencias, pues se puede tener una imagen real de lo que ocurre en el espacio epidural y cuando, además de adherencias, existen otros problemas como la inflamación o la aracnoiditis. Queda pues la incógnita sobre el futuro de la epidurolisis como una técnica a ser sustituida en los próximos años por la epiduroscopia, actualmente en fase de implantación en nuestro país, y de la que desconocemos cuáles son sus resultados y de la que carecemos de evidencias en su utilización. Quizás las múltiples variantes que ha tenido el protocolo original de Racz, en cuanto a la duración, dos días o uno, la utilización de los diferentes fármacos, con hialuronidasa o sin ella, con suero salino hipertónico o sin él, haya contribuido a la confusión y a las dudas sobre su realización, que han frenado la popularización de esta técnica, además de la utilización del suero salino hipertónico, en un espacio donde la comunicación con el espacio intradural es fácil. En el artículo que se publica en este número, se realiza un completo repaso de la fisiopatología, el diagnóstico y las diferentes técnicas publicadas con un exhaustivo repaso de la bibliografía, y en una segunda parte una descripción detallada del protocolo original de dos 
días con hialuronidasa, anestésicos locales y corticoides y suero salino hipertónico, que es el que sigue utilizando el Dr. Gabor Racz y que en sus expertas manos tiene una eficacia contrastada y con escasas complicaciones.

J. Insausti

\section{BIBLIOGRAFÍA}

1. Robertson JT. Role of peridural fibrosis in the failed back: A review. Eur Spine J 1996;5(Suppl 1):S2-6.

2. Manchikanti L, Rivera JJ, Pampati V, et al. One day lumbar epidural adhesiolysis and hypertonic saline neurolysis in treatment of chronic low back pain: A randomized, double-blind trial. Pain Physician 2004 Apr;7(2):177-86

3. Racz GB, Heavner JE, Trescot A. Percutaneous lysis of epidural adhesions--evidence for safety and efficacy. Pain Pract 2008;8(4):277-86.

4. Perkins WJ, Davis DH, Huntoon MA, Horlocker TT. A retained racz catheter fragment after epidural neurolysis: Implications during magnetic resonance imaging. Anesth Analg 2003 Jun;96(6):1717-9, table of contents.

5. Aldrete JA, Zapata JC, Ghaly R. Arachnoiditis following epidural adhesiolysis with hypertonic saline. Report of two cases. Pain Digest 1996;6:368-70.

6. Talu GK, Erdine S. Complications of epidural neuroplasty: A retrospective evaluation. Neuromodulation 2003;6(4):237-47. 